\title{
The COVID-19 crisis in South Africa: Protecting the vulnerable
}

As of 3 July 2020, global cases of COVID-19 surpassed 10 million with over half a million deaths in more than 210 countries. ${ }^{[1]}$ South Africa (SA) too reached a milestone last week, recording a total of 100000 laboratory-confirmed cases and 2500 deaths since March (as of 7 July 2020, total number of positive cases 215855 , total number of deaths 3 502). The epidemic's epicentre in the Western Cape, where a major surge has resulted in record hospital bed occupancy rates, is quickly being surpassed by Gauteng in the number of new cases. ${ }^{[2]}$ Similar trends in case numbers are being reported in Eastern Cape Province, stretching valiant healthcare workers in all three provinces. The remaining provinces are likely to follow suit.

While benefiting health service preparedness, continuation of SA's early lockdown was untenable owing to its severe socioeconomic effects and negative impacts on aspects of public health such as tuberculosis and vaccination programmes. However, its relaxation has increased social mixing, and the challenge we now face is how we as a country limit the surge in COVID-19 cases, especially in the most vulnerable at-risk populations, its impact on our health system, and the death rate from the infection.

Partial release of the lockdown has given many the impression that the dangers of COVID-19 have receded. This, together with what is essentially an 'invisible enemy', the need to reclaim some normality to our lives, the absolute essential need for many to return to work, travel in overcrowded transport and our social needs, will increase the risks of acquiring the coronavirus, and for a few, the risk of severe COVID-19. This will be most felt in the vulnerable groups, and it is essential that those at risk remain protected even if that means personal 'lockdowns'. So who comprise these groups, and how can they be protected?

We have learned much from international experience, ${ }^{[3]}$ but also from our own data collection. A sentinel hospital surveillance system (DATCOV ${ }^{[4]}$ ) was specially developed by the National Institute for Communicable Diseases in late March 2020 to report on epidemiological and clinical characteristics and outcome of patients hospitalised with COVID-19. ${ }^{[5]}$ It currently receives daily reports from 212 private and 75 public hospitals in SA and to date has received reports on 17693 patients for the period 5 March - 5 July 2020. ${ }^{[5]}$ The following are associated with severe COVID-19 and fatal outcome in SA: older age groups; male sex; and having comorbid hypertension, diabetes, chronic cardiac disease, chronic lung disease, chronic renal disease, malignancy, HIV, active tuberculosis and obesity. ${ }^{[6]}$ The highest risk is from malignancy (adjusted odds ratio (aOR) 2.8), chronic renal disease (aOR 1.9), chronic cardiac disease (aOR 1.8) and active tuberculosis (aOR 1.8), with modest risk associated with hypertension (aOR 1.3), diabetes (aOR 1.5) and HIV (aOR 1.4).

These comorbidities are very similar to those published from observational studies in many countries in the Northern Hemisphere. ${ }^{[7,8]}$ Of hospitalised patients with no comorbidities, $199 / 3144(6.3 \%)$ died, of those with one comorbid condition, $588 / 3756$ (15.7\%) died, of those with two comorbid conditions, $576 / 2554$ (22.6\%) died, and of those with three or more comorbid conditions, 402/1 422 (28.3\%) died.

Data from a well-characterised cohort in the Western Cape and from the DATCOV system showed that individuals living with HIV have a modest 1.4 - 2-fold increase in risk of COVID-19-associated death compared with HIV-uninfected persons. ${ }^{[5]}$ Despite fears that HIV-positive people with advanced immunosuppression would have very high mortality rates, there was no evidence of an association between increasing immunosuppression and severity of COVID-19 in the Western Cape data. Importantly, many HIV-positive people had other comorbidities (tuberculosis, diabetes, hypertension, etc.) that put them at increased risk of severe COVID-19-related illness when taken together.

So how can we reduce risks in these vulnerable groups? Firstly, personal protection is key. Those of advanced age and/or with significant comorbidities should socially distance as much as possible. Asymptomatic or minimally symptomatic SARS-CoV-2 infections are common, and crowded areas abound. Social and religious gatherings and various leisure and sporting activities pose a high risk to the vulnerable and should be avoided as much as possible. In addition to physically distancing the vulnerable from possible harm, non-pharmaceutical measures to reduce virus transmission must be meticulously adhered to, i.e. wearing a cloth mask whenever in public, hand hygiene, and regular cleaning of commonly used surfaces in the home and at work. Currently, these are the only means we have of limiting transmission.

Second is for persons with comorbidities to work with their healthcare providers to optimise control of that illness, e.g. improving diabetic or blood pressure control, ensuring adherence with medications, e.g. antiretrovirals or tuberculosis drugs, etc. Early identification of those with comorbidities and COVID-19 who decompensate and require hospitalisation is key for early and urgent oxygen therapy and consideration for dexamethasone. Medical personnel $\geq 60$ years of age and with any related comorbidities should not work in the front line in roles that increase the risk of COVID-19 acquisition, and can use their considerable experience and knowledge in a different but essential support role.

While the first wave and major surges of COVID-19 cases will pass, the likely scenario, and evidence from countries in the Northern Hemisphere, is that COVID-19 will be with us for a long time yet and associated with clusters, outbreaks, and even a second wave. In the absence of an effective vaccine, vulnerable groups need to be protected.

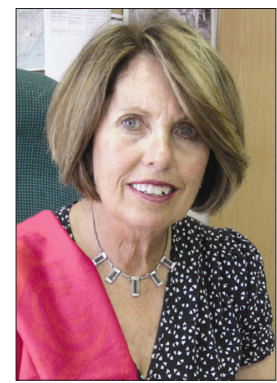

Lucille Blumberg

National Institute for Communicable Diseases of the National Health Laboratory Service, Johannesburg, and the University of Pretoria, South Africa lucilleb@nicd.ac.za

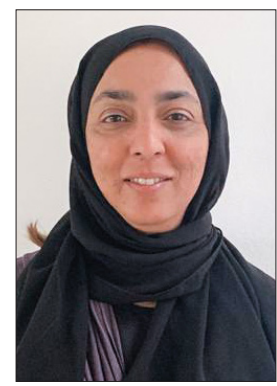

Waasila Jassat National Institute for Communicable Diseases of the National Health Laboratory Service, Johannesburg, South Africa 


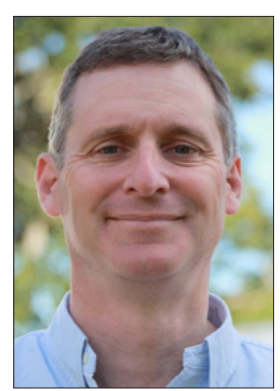

Marc Mendelson

Head of Infectious Diseases and HIV Medicine, Groote Schuur Hospital and Faculty of Health Sciences, University of Cape Town, South Africa

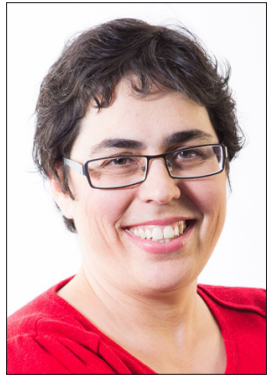

\section{Cheryl Cohen}

National Institute for Communicable Diseases of the National Health Laboratory Service, Johannesburg, South Africa
1. World Health Organization. Situation report - 165, Coronavirus disease 2019 (COVID-19). 3 July 2020. https://www.who.int/emergencies/diseases/novel-coronavirus-2019/situation-reports (accessed 3 July 2020).

2. National Institute for Communicable Diseases. COVID-19 weekly epidemiology brief. Week 26, 2020. https://www.nicd.ac.za/wp-content/uploads/2020/07/NICD-Weekly-Epidemiological-Brief_-Weekending-27-June-2020-Final.pdf (accessed 8 July 2020).

3. Docherty AB, Harrison EM, Green CA, et al.; ISARIC4C investigators. Features of 20133 UK patients in hospital with COVID-19 using the ISARIC WHO Clinical Characterisation Protocol: Prospective observational cohort study. BMJ 2020;369:m1985. https://doi.org/10.1136/bmj.m1985

4. Jassat W, Cohen C, Kufa T, et al. DATCOV: A sentinel surveillance programme for hospitalised individuals with COVID-19 in South Africa, 2020. COVID-19 Special Public Health Surveillance Bulletin, Vol. 18, Supplementary Issue 1, 10 June 2020. https://www.nicd.ac.za/wp-content/ uploads/2020/06/COVID-19-Special-Public-Health-Surveillance-Bulletin-10-June-2020-005.pdf (accessed 8 July 2020).

5. National Institute for Communicable Diseases. National COVID-19 daily report. 7 July 2020. https:// www nicd pdf (accessed 8 July 2020).

National Institute for Communicable Diseases. NICD COVID-19 surveillance in selected hospitals. 7 July 2020. https://www.nicd.ac.za/wp-content/uploads/2020/07/NICD-COVID-19-Daily-SentinelHospital-Surveillance-report-National-20200707.pdf (accessed 8 July 2020).

7. National Institute for Communicable Diseases. COVID-19 sentinel hospital surveillance update. Week 7. National Institute for Communicable Diseases. COVID-19 sentinel hospital surveillance update. Week
26, 2020. https://www.nicd.ac.za/wp-content/uploads/2020/07/NICD-COVID-19-Weekly-Sentinel26, 2020. https://www.nicd.ac.za/wp-content/uploads/2020/07/NICD-COVID-19-W

Hospital-Surveillnace-update-Week-26-2020-updated.pdf (accessed 8 July 2020).

8. Davies M, Boulle A. Risk of COVID-19 death among people with HIV: A population cohort analysis
from the Western Cape Province, South Africa. COVID-19 Special Public Health Surveillance Bulletin, from the Western Cape Province, South Africa. COVID-19 Special Public Health Surveillance Bulletin,
Vol. 18, Supplementary Issue 2, 22 June 2020. https://www.nicd.ac.za/wp-content/uploads/2020/06/ COVID-19-Special-Public-Health-Surveillance-Bulletin-22-June-2020.pdf (accessed 8 July 2020).

S Afr Med J 2020;110(9):825-826. https://doi.org/10.7196/SAMJ.2020.v110i9.15116 